3D MPRAGE MRI were performed at study entry and 12 and 24 months later in 45 subjects with MCI. Hippocampal volume (HV) was assessed with Neuroquant ${ }^{\circledR}$. The age adjusted 25 th percentile was used to define hippocampal atrophy (HA). A b burden was quantified using SUVR using the cerebellar cortex as reference region. Cut-off for low vs high SUVR was 1.4. Cox Proportional Hazards were used to calculate relative risk (RR) corrected for age, gender and years of education. Results: At baseline $53 \%$ of the MCI participants had high neocortical FBB binding while $76 \%$ had hippocampal atrophy. Composite memory scores correlated with both neocortical SUVR $(\mathrm{r}=-0.60, P<0.0001)$ and HV $(\mathrm{r}=0.39, P=0.02)$. At 24-month follow-up, increase in neocortical SUVR was observed in MCI with high-FBB $(+3.1 \%, P=0.02)$. Progression to AD occurred in $75 \%$ of MCI with high-FBB (RR $10.9 P<0.001)$ and $53 \%$ of MCI with HA (RR 4.1 $P=0.03$ ). HA lost significance in multivariate analysis. Of MCI with both high FBB and HA, 80\% progressed to AD. Of the lowFBB MCI, 19\% progressed to other dementias. For progression to any dementia, HA had a predictive RR 5.0, $P=0.009$. Conclusions: High $18 \mathrm{~F}$ -Florbetaben binding indicates a very high risk of progression from MCI to Alzheimer's disease within two years (RR 10.9) and was a stronger and more specific risk factor than hippocampal atrophy in this cohort.

\section{IC-P-032 CROSS-SECTIONAL CEREBRAL VOLUMETRIC DIFFERENCES AND ASSOCIATIONS WITH ESTIMATED TIME TO AGE-AT-ONSET IN FAMILIAL ALZHEIMER'S DISEASE: FINDINGS FROM THE DIAN STUDY}

Kirsi Kinnunen ${ }^{1}$, David Cash ${ }^{2}$, Yuying Liang ${ }^{3}$, Kelvin Leung ${ }^{3}$, Manuel Cardoso ${ }^{3}$, Marc Modat ${ }^{3}$, Ian Malone ${ }^{3}$, Tom Yeatman ${ }^{3}$, Jennifer Nicholas ${ }^{3}$, Tammie Benzinger ${ }^{4}$, Robert Koeppe ${ }^{5}$, Clifford Jack ${ }^{6}$, Marcus Raichle ${ }^{7}$, Daniel Marcus ${ }^{8}$, John Ringman ${ }^{9}$, Paul Thompson ${ }^{9}$, Andrew Saykin ${ }^{10}$, Bernardino Ghetti ${ }^{10}$, Stephen Salloway ${ }^{11}$, Stephen Correia $^{12}$, Keith Johnson ${ }^{13}$, Reisa Sperling ${ }^{14}$, Peter Schofield ${ }^{15}$, Christopher Rowe ${ }^{16}$, Colin Masters ${ }^{17}$, Victor Villemagne ${ }^{16}$, Adam Brickman ${ }^{18}$, Richard Mayeux ${ }^{19}$, Ralph Martins ${ }^{20}$, Michael Weiner ${ }^{21}$, Randall Bateman ${ }^{4}$, Alison Goate ${ }^{22}$, Virginia Buckles ${ }^{23}$, Krista Moulder ${ }^{8}$, John Morris ${ }^{23}$, Martin Rossor ${ }^{3}$, Sebastien Ourselin ${ }^{3}$, Nick Fox ${ }^{3},{ }^{1}$ Dementia Research Centre, University College of London, Institute of Neurology, London, United Kingdom; ${ }^{2}$ University College of London, London, United Kingdom; ${ }^{3}$ University College of London, London, United Kingdom; ${ }^{4}$ Washington University School of Medicine, St. Louis, Missouri, United States; ${ }^{5}$ University of Michigan Health System, Ann Arbor, Michigan, United States; ${ }^{6}$ Mayo Clinic, Rochester, Minnesota, United States; ${ }^{7}$ Washington University School of Medicine, St. Louis, Missouri, United States; ${ }^{8}$ Washington University in St. Louis, St. Louis, Missouri, United States; ${ }^{9}$ UCLA, Los Angeles, California, United States; ${ }^{10}$ Indiana University School of Medicine, Indianapolis, Indiana, United States; ${ }^{11}$ Brown University, Providence, Rhode Island, United States; ${ }^{12}$ Brown Medical School, Providence, Rhode Island, United States; ${ }^{13}$ Massachusetts General Hospital, Boston, Massachusetts, United States; ${ }^{14}$ Harvard Medical School, Boston, Massachusetts, United States; ${ }^{15}$ Neuroscience Research Australia, Randwick-Sydney, Australia; ${ }^{16}$ Austin Health, Melbourne, Australia; ${ }^{17}$ University of Melbourne, Melbourne, Victoria, Australia, ${ }^{18}$ Columbia University, New York, New York, United States; ${ }^{19}$ Columbia University Medical Center, New York, New York, United States; ${ }^{20}$ Edith Cowan University, Perth, Australia; ${ }^{21}$ University of California San Francisco, San Francisco, California, United States; ${ }^{22}$ Washington University in St. Louis, St. Louis, Missouri, United States; ${ }^{23}$ Washington University, St. Louis, Missouri, United States.

Background: A key challenge for Alzheimer's disease (AD) research is to identify the most reliable markers of early disease progression. Autosomal dominant $\mathrm{AD}$ provides a unique opportunity to study the earliest changes. We assessed cross-sectional volumes of whole brain, ventricles, and hippocampi within the Dominantly Inherited Alzheimer Network (DIAN) cohort. Methods: 158 participants were included: 55 of whom were non-carriers
(NC); 59 were asymptomatic carriers (aMut+) with Clinical Dementia Rating (CDR) of 0 ; and 44 were symptomatic carriers (sMut+) with $\mathrm{CDR}>0$. All participants underwent volumetric T1-weighted MRI. Automated segmentation techniques were used to delineate the whole brain, ventricles, and hippocampi. All volumes were adjusted for total intracranial volume and age. Ventricular and hippocampal volumes were additionally adjusted for sex. The relationships with time to expected age-at-onset, estimated from affected parent's age-at-onset, were also examined. Results: Statistically significant $(P<0.001)$ differences were observed in brain, ventricles and hippocampi between the sMut+ and both NC and aMut+. There were no significant differences between the aMut+ and the $\mathrm{NC}$, although a trend was observed for whole-brain volumes. The Figure shows average corrected volumes in each group. In addition, the aMut+ showed significant $(P<0.05)$ correlations between time to expected age-at-onset and whole-brain and ventricular volumes. For the sMut+, similar significant correlations $(P$ $<0.05$ ) were observed between the predicted age-at-onset and whole-brain and hippocampal volumes. Conclusions: Participants with dementia already had significantly greater atrophy: smaller brain and hippocampal volumes and larger ventricles. Unaffected carriers did not show significant differences compared to non-carriers: this overlap may be due to the wide normal range in regional brain volumes, and the inclusion of carriers who are many years before expected age-at-onset. Notably there was a significant association between estimated age-at-onset and brain volume in asymptomatic carriers suggesting atrophy increases (possibly non-linearly) prior to symptoms. Longitudinal measurements of atrophy may be a more sensitive biomarker than cross-sectional measures: the ongoing follow-up of DIAN subjects will allow this to be assessed.
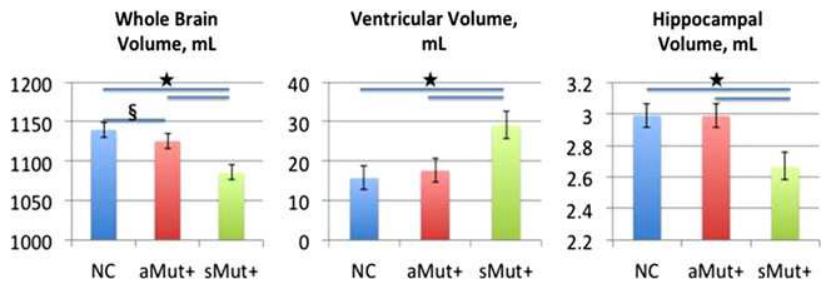

Figure 1. Average volumes (error bars represent $95 \%$ confidence intervals) for the structures of interest. All volumes have been adjusted for covariates of Total Intracranial Volume and Age. The Ventricles and Hippocampi have additionally been adjusted for gender. *indicates $P<0.001$. $-\S$ indicates $(P=0.075)$ between aMut+ brain volume vs. NC brain volume.

\section{IC-P-034 DEFINITION OF HARMONIZED PROTOCOL FOR HIPPOCAMPAL SEGMENTATION}

Marina Boccardi ${ }^{1}$, Martina Bocchetta ${ }^{2}$, Liana Apostolova ${ }^{3}$, Josephine Barnes ${ }^{4}$, George Bartzokis ${ }^{5}$, Gabriele Corbetta ${ }^{2}$, Charles DeCarli ${ }^{6}$, Leyla DeToledo-Morrell ${ }^{7}$, Michael Firbank ${ }^{8}$, Rossana Ganzola ${ }^{2}$, Lotte Gerritsen ${ }^{9}$, Wouter Henneman ${ }^{10}$, Ronald Killiany ${ }^{11}$, Nikolai Malykhin ${ }^{12}$, Patrizio Pasqualetti ${ }^{13}$, Jens Pruessner ${ }^{14}$, Alberto Redolfi ${ }^{2}$, Nicolas Robitaille ${ }^{15}$, Hilkka Soininen ${ }^{16}$, Daniele Tolomeo $^{2}$, Lei Wang ${ }^{17}$, Craig Watson ${ }^{18}$, Henrike Wolf ${ }^{19}$, Simon Duchesne ${ }^{15}$, Clifford Jack ${ }^{20}$, Giovanni Frisoni ${ }^{21},{ }^{1}$ IRCCS S. Giovanni di Dio - Fatebenefratelli, Brescia, Italy; ${ }^{2}$ IRCCS S. Giovanni di Dio Fatebenefratelli, Brescia, Italy; ${ }^{3}$ David Geffen School of Medicine, University of California, Los Angeles, California, United States; ${ }^{4}$ UCL Institute of Neurology, University College of London, London, United Kingdom; ${ }^{5}$ David Geffen School of Medicine at UCLA, Los Angeles, California, United States; ${ }^{6}$ University of California at Davis, Sacramento, California, United States; ${ }^{7}$ Rush University, Chicago, Illinois, United States; ${ }^{8}$ Institute for Ageing and Health, Newcastle University, Wolfson Research Centre, Newcastle upon Tyne, United Kingdom; ${ }^{9}$ Karolinska Institute, Stockholm, Sweden; ${ }^{10}$ VU University Medical Center, Amsterdam, Netherlands; ${ }^{11}$ Boston University School of Medicine, Boston, Massachusetts, United States; ${ }^{2}$ Centre for Neuroscience, University of Alberta, Edmonton, Alberta, Canada; ${ }^{13}$ Associazione Fatebenefratelli per 